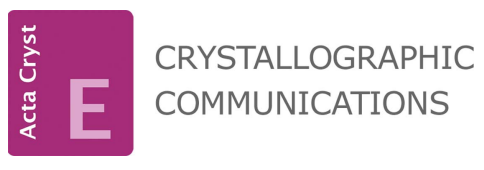

ISSN 2056-9890

Received 10 September 2019

Accepted 22 September 2019

Edited by W. T. A. Harrison, University of Aberdeen, Scotland

Keywords: crystal structure; charge-assisted hydrogen bonding; thiazolidine ring; Hirshfeld surface analysis.

CCDC reference: 1955268

Supporting information: this article has supporting information at journals.iucr.org/e

\section{Crystal structure and Hirshfeld surface analysis of 3-amino-5-phenylthiazolidin-2-iminium bromide}

\author{
Gulnara Sh. Duruskari, ${ }^{a}$ Ali N. Khalilov, ${ }^{\text {a,b }}$ Mehmet Akkurt, ${ }^{\mathrm{c}}$ Gunay Z. \\ Mammadova, ${ }^{a}$ Taras Chyrka $^{\mathrm{d} *}$ and Abel M. Maharramov ${ }^{\mathrm{a}}$
}

\begin{abstract}
${ }^{a}$ Organic Chemistry Department, Baku State University, Z. Xalilov str. 23, Az, 1148 Baku, Azerbaijan, ${ }^{\mathbf{b}}$ Department of Physics and Chemistry, "Composite Materials" Scientific Research Center, Azerbaijan State Economic University (UNEC), H. Aliyev str. 135, Az 1063, Baku, Azerbaijan, 'Department of Physics, Faculty of Sciences, Erciyes University, 38039 Kayseri, Turkey, and ${ }^{\mathbf{d}}$ Department of Theoretical and Industrial Heat Engineering (TPT), National Technical University of Ukraine, "Igor Sikorsky Kyiv Polytechnic Institute", 03056, Kyiv, Ukraine. *Correspondence e-mail: mustford@ukr.net
\end{abstract}

In the cation of the title salt, $\mathrm{C}_{9} \mathrm{H}_{12} \mathrm{~N}_{3} \mathrm{~S}^{+} \cdot \mathrm{Br}^{-}$, the thiazolidine ring adopts an envelope conformation with the $\mathrm{C}$ atom adjacent to the phenyl ring as the flap. In the crystal, $\mathrm{N}-\mathrm{H} \cdots \mathrm{Br}$ hydrogen bonds link the components into a threedimensional network. Weak $\pi-\pi$ stacking interactions between the phenyl rings of adjacent cations also contribute to the molecular packing. A Hirshfeld surface analysis was conducted to quantify the contributions of the different intermolecular interactions and contacts.

\section{Chemical context}

As well as their synthetic utility, thiazolidine derivatives possess a broad spectrum of biological activities such as antimalarial, antibacterial, antimicrobial, anti-inflammatory, anticancer, etc. The biological activities of compounds containing a thiazolidine core, such as 1,3-thiazolidines, 2,4dione-, 4-oxo-thiazolidine, etc. were summarized in a recent review (Makwana \& Malani, 2017). On the other hand, as hydrazones these $\mathrm{N}$-containing ligands have been widely used in the synthesis of coordination compounds (Gurbanov et al., $2018 a, b)$. The non-covalent donor or acceptor properties of $\mathrm{N}$ containing ligands can also contribute to their catalytic activity, among other properties (Mahmudov et al., 2019; Zubkov et al., 2018). As part of our ongoing work in this area, we now describe the synthesis and structure of the title molecular salt, $\mathrm{C}_{9} \mathrm{H}_{12} \mathrm{~N}_{3} \mathrm{~S}^{+} \cdot \mathrm{Br}^{-}$, (I).<smiles>NN1CC(c2ccccc2)SC1=[NH2+]</smiles>

\section{Structural commentary}

In the cation of (I) (Fig. 1), the thiazolidine ring (S1/N1/C1C3) adopts an envelope conformation with puckering parameters of $Q(2)=0.317(2) \AA$ and $\varphi(2)=225.2(4)^{\circ}$ : the flap atom is $\mathrm{C} 1$. In the arbitrarily chosen asymmetric unit, $\mathrm{C} 1$ has an $R$ configuration, but symmetry generates a racemic mixture 
Table 1

Hydrogen-bond geometry $\left(\AA,^{\circ}\right)$.

\begin{tabular}{lllll}
\hline$D-\mathrm{H} \cdots A$ & $D-\mathrm{H}$ & $\mathrm{H} \cdots A$ & $D \cdots A$ & $D-\mathrm{H} \cdots A$ \\
\hline $\mathrm{N} 2-\mathrm{H} 2 A \cdots \mathrm{Br} 1^{\mathrm{i}}$ & 0.90 & 2.68 & $3.530(2)$ & 158 \\
$\mathrm{~N} 2-\mathrm{H} 2 B \cdots \mathrm{Br} 1^{\mathrm{ii}}$ & 0.90 & 2.73 & $3.524(2)$ & 148 \\
$\mathrm{~N} 3-\mathrm{H} 3 A \cdots \mathrm{Br} 1$ & 0.90 & 2.38 & $3.271(2)$ & 169 \\
$\mathrm{~N} 3-\mathrm{H} 3 B \cdots \mathrm{Br} 1^{\mathrm{iii}}$ & 0.90 & 2.56 & $3.337(2)$ & 145 \\
\hline
\end{tabular}

Symmetry codes: (i) $\quad-x+1,-y+1,-z+1 ; \quad$ (ii) $\quad x+\frac{1}{2},-y+\frac{3}{2}, z+\frac{1}{2}$; (iii) $-x+\frac{1}{2}, y+\frac{1}{2},-z+\frac{1}{2}$.

in the crystal. The dihedral angle between the mean plane of the thiazolidine ring (all atoms) and the phenyl ring (C4-C9) is $89.27(13)^{\circ}$.

\section{Supramolecular features and Hirshfeld surface analysis}

In the crystal, each cation forms $\mathrm{N}-\mathrm{H} \cdots \mathrm{Br}$ hydrogen bonds (Table 1) as well as aromatic $\pi-\pi$ stacking interactions between the phenyl rings of adjacent cations $\left[C g 2 \cdots C g 2^{\text {iv }}=\right.$ 3.7758 (16) $\AA$; symmetry code: (iv) $1-x, 1-y, 2-z$; where $C g 2$ is the centroid of the phenyl ring of the cation]: chains of cations form along the [101] direction (Fig. 2). Taking into account the hydrogen bonding and $\pi-\pi$ stacking, the overall connectivity is three-dimensional.

Hirshfeld surface analysis (Spackman \& Jayatilaka, 2009; Spackman \& McKinnon, 2002) was carried out with CrystalExplorer3.1 (Wolff et al., 2012) to further investigate the presence of hydrogen bonds and intermolecular interactions in the crystal structure (see supporting information). Fig. 3(a) shows the two-dimensional fingerprint of the sum of the contacts contributing to the Hirshfeld surface represented in normal mode while those delineated into $\mathrm{H} \cdots \mathrm{H}(41.5 \%)$, $\mathrm{Br} \cdots \mathrm{N} / \mathrm{N} \cdots \mathrm{Br}(24.1 \%), \mathrm{C} \cdot \mathrm{H} / \mathrm{H} \cdots \mathrm{C}(13.8 \%)$ and $\mathrm{S} \cdot \mathrm{H} /$ $\mathrm{H} \cdots \mathrm{S}(11.7 \%)$ contacts, respectively, are shown in Fig. $3 b-e$. All contacts are listed in Table 2.

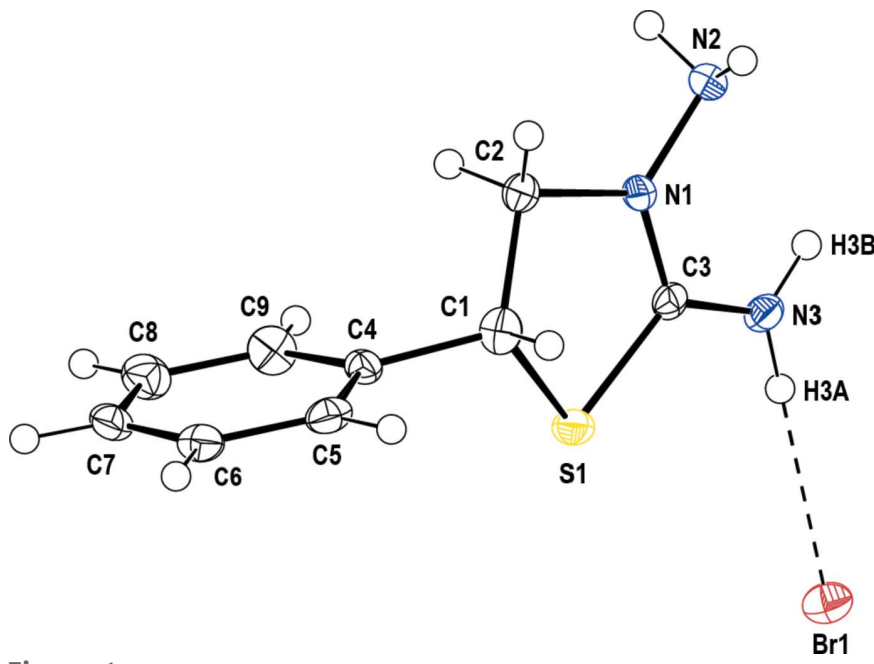

Figure 1

The molecular structure of the title salt. Displacement ellipsoids are drawn at the $50 \%$ probability level and the $\mathrm{H} \cdots \mathrm{Br}$ hydrogen bond is indicated by a dashed line.
Table 2

Percentage contributions of interatomic contacts to the Hirshfeld surface for the title salt.

\begin{tabular}{ll}
\hline Contact & Percentage contribution \\
\hline $\mathrm{H} \cdots \mathrm{H}$ & 41.5 \\
$\mathrm{Br} \cdots \mathrm{N} / \mathrm{N} \cdots \mathrm{Br}$ & 24.1 \\
$\mathrm{C} \cdots \mathrm{H} / \mathrm{H} \cdots \mathrm{C}$ & 13.8 \\
$\mathrm{~S} \cdots \mathrm{H} / \mathrm{H} \cdots \mathrm{S}$ & 11.7 \\
$\mathrm{~N} \cdots \mathrm{H} / \mathrm{H} \cdots \mathrm{N}$ & 3.6 \\
$\mathrm{C} \cdots \mathrm{C}$ & 3.3 \\
$\mathrm{~N} \cdots \mathrm{C} / \mathrm{C} \cdots \mathrm{N}$ & 1.5 \\
$\mathrm{~N} \cdots \mathrm{N}$ & 0.3 \\
$\mathrm{~S} \cdots \mathrm{C} / \mathrm{C} \cdots \mathrm{S}$ & 0.3 \\
\hline
\end{tabular}

\section{Database survey}

A search of the Cambridge Structural Database (CSD, Version 5.40, February 2019; Groom et al., 2016) for 2-thiazolidiniminium compounds gave eight hits, viz. BOBWIB (Khalilov et al., 2019), UDELUN (Akkurt et al., 2018), WILBIC (Marthi et al., 1994), WILBOI (Marthi et al., 1994), WILBOI01 (Marthi et al., 1994), YITCEJ (Martem'yanova et al., 1993a), YITCAF (Martem'yanova et al., 1993b) and YOPLUK (Marthi et al., 1995).

In the crystal of BOBWIB (Khalilov et al., 2019), the thiazolidine ring adopts an envelope conformation. In the crystal, centrosymmetrically related cations and anions are linked into dimeric units via $\mathrm{N}-\mathrm{H} \cdots \mathrm{Br}$ hydrogen bonds, which are further connected by weak $\mathrm{C}-\mathrm{H} \cdots \mathrm{Br}$ hydrogen bonds into chains parallel to [110]. In the crystal of UDELUN (Akkurt et al., 2018), $\mathrm{C}-\mathrm{H} \cdots \mathrm{Br}$ and $\mathrm{N}-\mathrm{H} \cdots \mathrm{Br}$ hydrogen bonds link the components into a three-dimensional network with the cations and anions stacked along the $b$-axis direction. Weak $\mathrm{C}-\mathrm{H} \cdots \pi$ interactions, which only involve the minor disorder component of the ring, also contribute to the molecular packing. In

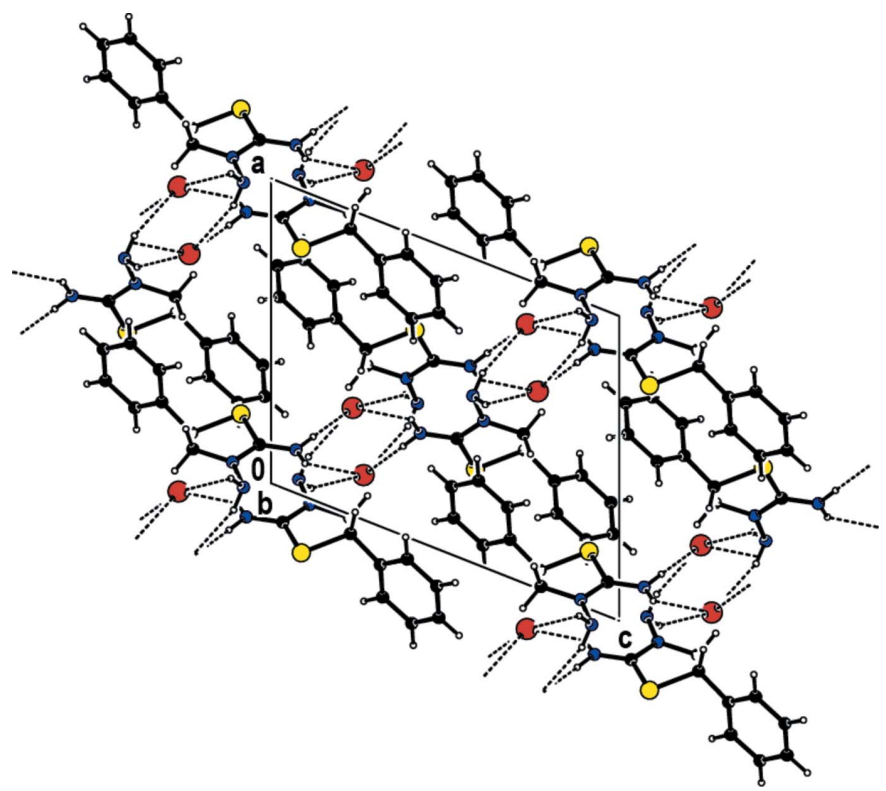

Figure 2

Part of the crystal structure of the title compound, showing the formation of $\mathrm{N}-\mathrm{H} \cdots \mathrm{Br}$ hydrogen bonds in the ac plane. 
addition, there are also inversion-related $\mathrm{Cl} \cdots \mathrm{Cl}$ halogen bonds and $\mathrm{C}-\mathrm{Cl} \cdots \pi$ (ring) contacts. In the other structures, the 3-N atom carries a $\mathrm{C}$ substituent: the first three crystal structures were determined for racemic (WILBIC; Marthi et al., 1994) and two optically active samples (WILBOI and WILBOI01; Marthi et al., 1994) of 3-(2'-chloro-2'-phenylethyl)-2-thiazolidiniminium $p$-toluenesulfonate. In all three structures, the most disordered fragment of these molecules is the asymmetric $\mathrm{C}$ atom and the $\mathrm{Cl}$ atom attached to it. The disorder of the cation in the racemate corresponds to the presence of both enantiomers at each site in the ratio 0.821 (3): 0.179 (3). The system of hydrogen bonds connecting two cations and two anions into 12-membered rings is identical in the racemic and in the optically active crystals. YITCEJ (Martem'yanova et al., 1993a) is a product of the interaction of 2-amino-5-methylthiazoline with methyl iodide, with alkylation at the endocylic nitrogen atom, while YITCAF

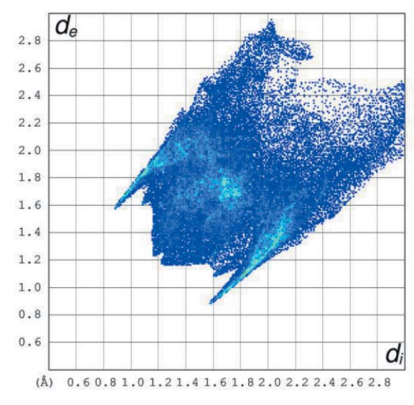

(a) All...All

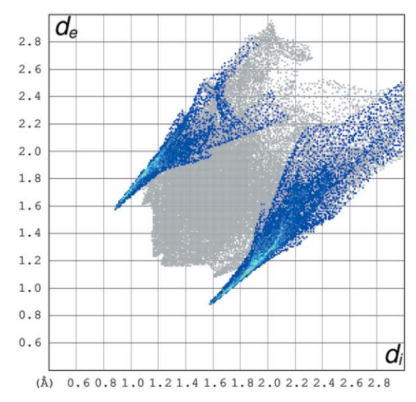

(c) Br...N / N...Br

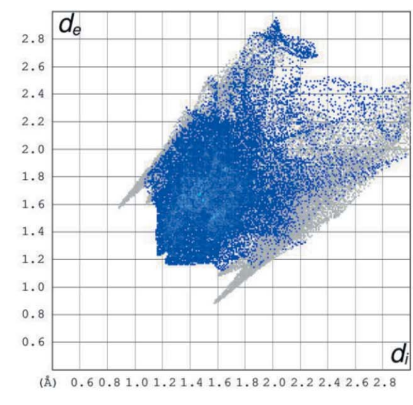

(b) $\mathbf{H}$...H

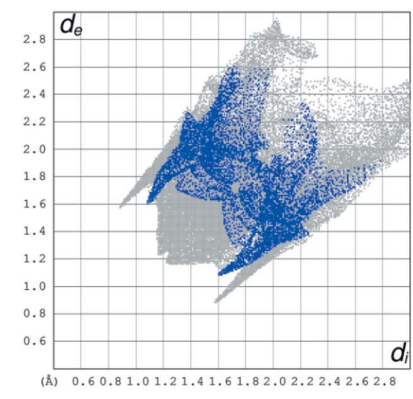

(d) C...H / H...C

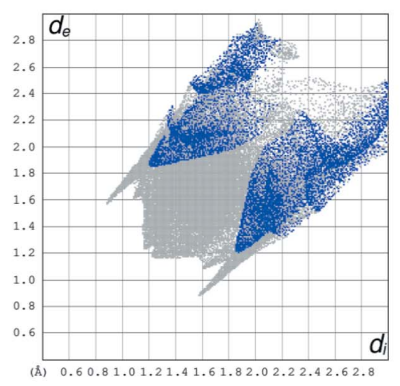

(e) S...H / H...S

Figure 3

The two-dimensional fingerprint plots of the title salt, showing (a) all interactions, and delineated into $(b) \mathrm{H} \cdots \mathrm{H},(c) \mathrm{Br} \cdots \mathrm{N} / \mathrm{N} \cdots \mathrm{Br},(d)$ $\mathrm{C} \cdots \mathrm{H} / \mathrm{H} \cdots \mathrm{C}$ and $(e) \mathrm{S} \cdots \mathrm{H} / \mathrm{H} \cdots \mathrm{S}$ interactions $\left[d_{\mathrm{e}}\right.$ and $d_{\mathrm{i}}$ represent the distances from a point on the Hirshfeld surface to the nearest atoms outside (external) and inside (internal) the surface, respectively].

Table 3

Experimental details.

Crystal data

Chemical formula

$M_{\mathrm{r}}$

Crystal system, space group

Temperature (K)

$a, b, c(\AA)$

$\beta\left({ }^{\circ}\right)$

$V\left(\AA^{3}\right)$

$Z$

Radiation type

$\mu\left(\mathrm{mm}^{-1}\right)$

Crystal size (mm)

Data collection

Diffractometer

Absorption correction

$T_{\min }, T_{\max }$

No. of measured, independent and observed $[I>2 \sigma(I)]$ reflections

$R_{\text {in }}$

$(\sin \theta / \lambda)_{\max }\left(\AA^{-1}\right)$

Refinement

$R\left[F^{2}>2 \sigma\left(F^{2}\right)\right], w R\left(F^{2}\right), S$

No. of reflections

No. of parameters

$\mathrm{H}$-atom treatment

$\Delta \rho_{\max }, \Delta \rho_{\min }\left(\mathrm{e} \AA^{-3}\right)$

Computer programs: APEX2 and SAINT (Bruker, 2003), SHELXT2014 (Sheldrick, 2015a), SHELXL2016 (Sheldrick, 2015b), ORTEP-3 for Windows (Farrugia, 2012) and PLATON (Spek, 2003).

(Martem'yanova et al., 1993b) is a product of the reaction of 3-nitro-5-methoxy-, 3-nitro-5-chloro-, and 3-bromo-5-nitrosalicylaldehyde with the heterocyclic base to form the salt-like complexes.

\section{Synthesis and crystallization}

To a solution of $2.2 \mathrm{mmol}(0.6 \mathrm{~g})$ (1,2-dibromoethyl)benzene in $20 \mathrm{ml}$ of ethanol were added $2.3 \mathrm{mmol}(0.3 \mathrm{~g})$ of thiosemicarbazide hydrochloride; 3-4 drops of piperidine were added and the mixture was refluxed for $7 \mathrm{~h}$. The reaction mixture was cooled to room temperature and the solid product was precipitated from solution, collected by filtration and recrystallized from ethanol solution to give colourless crystals of (I) with a yield of $88 \%$, m.p. $=468 \mathrm{~K}$. Analysis calculated for $\mathrm{C}_{9} \mathrm{H}_{12} \mathrm{BrN}_{3} \mathrm{~S}$ : C 39.43; H 4.41; N 15.33. Found: C 39.40; H 4.39; $\mathrm{N} 15.30 \% .{ }^{1} \mathrm{H}$ NMR $\left(300 \mathrm{MHz}, \mathrm{DMSO}-d_{6}\right): 4.16(q, 1 \mathrm{H}$, $\left.\mathrm{CH}_{2},{ }^{3} J_{\mathrm{H}-\mathrm{H}}=5.4\right) ; 4.45\left(t, 1 \mathrm{H}, \mathrm{CH}_{2},{ }^{3} J_{\mathrm{H}-\mathrm{H}}=8.4\right) ; 5.25(t, 1 \mathrm{H}$, $\left.\mathrm{CH}-\mathrm{Ar},{ }^{3} J_{\mathrm{H}-\mathrm{H}}=5.4\right) ; 7.32-7.50(m, 5 \mathrm{H}, 5 \mathrm{Ar}-\mathrm{H}) ; 9.12(s, 2 \mathrm{H}$, $\left.\mathrm{NH}_{2}\right) ; 9,78(s, 1 \mathrm{H}, \mathrm{NH}=) .{ }^{13} \mathrm{C}$ NMR $\left(75 \mathrm{MHz}, \mathrm{DMSO}-d_{6}\right)$ : 44.42, 62.06, 127.59, 128.76, 129.17, 138.85, 168.53. MS (ESI), $m / z: 194.28\left[\mathrm{C}_{9} \mathrm{H}_{12} \mathrm{~N}_{3} \mathrm{~S}\right]^{+}$and $79.88 \mathrm{Br}^{-}$.

\section{Refinement}

Crystal data, data collection and structure refinement details are summarized in Table 3. All $\mathrm{H}$ atoms on $\mathrm{C}$ atoms were placed at calculated positions $(\mathrm{C}-\mathrm{H}=0.95-1.00 \AA)$ and 
refined using a riding model. The $\mathrm{N}$-bound hydrogen atoms were located from difference-Fourier maps and relocated to idealized locations $(\mathrm{N}-\mathrm{H}=0.90 \AA)$ and refined as riding atoms. The constraint $U_{\text {iso }}(\mathrm{H})=1.2 U_{\text {eq }}$ (carrier) was applied in all cases. One outlier (101) was omitted in the final cycles of refinement.

\section{Acknowledgements}

ANK is grateful to Baku State University for the "50+50" individual grant in support of this work.

\section{Funding information}

ANK is grateful to Baku State University for the "50+50" individual grant insupport of this work.

\section{References}

Akkurt, M., Duruskari, G. S., Toze, F. A. A., Khalilov, A. N. \& Huseynova, A. T. (2018). Acta Cryst. E74, 1168-1172.

Bruker (2003). APEX2, SAINT and SADABS. Bruker AXS Inc., Madison, Wisconsin, USA.

Farrugia, L. J. (2012). J. Appl. Cryst. 45, 849-854.

Groom, C. R., Bruno, I. J., Lightfoot, M. P. \& Ward, S. C. (2016). Acta Cryst. B72, 171-179.

Gurbanov, A. V., Huseynov, F. E., Mahmoudi, G., Maharramov, A. M., Guedes da Silva, F. C., Mahmudov, K. T. \& Pombeiro, A. J. L. (2018a). Inorg. Chim. Acta, 469, 197-201.
Gurbanov, A. V., Maharramov, A. M., Zubkov, F. I., Saifutdinov, A. M. \& Guseinov, F. I. (2018b). Aust. J. Chem. 71, 190-194.

Khalilov, A. N., Atioğlu, Z., Akkurt, M., Duruskari, G. S., Toze, F. A. A. \& Huseynova, A. T. (2019). Acta Cryst. E75, 662-666.

Mahmudov, K. T., Gurbanov, A. V., Guseinov, F. I. \& Guedes da Silva, M. F. C. (2019). Coord. Chem. Rev. 387, 32-46.

Makwana, H. R. \& Malani, A. H. (2017). IOSR J. Appl. Chem. 10, 7684.

Martem'yanova, N. A., Chunaev, Y. M., Przhiyalgovskaya, N. M., Kurkovskaya, L. N., Filipenko, O. S. \& Aldoshin, S. M. (1993a). Khim. Geterotsikl. Soedin. pp. 415-419.

Martem'yanova, N. A., Chunaev, Y. M., Przhiyalgovskaya, N. M., Kurkovskaya, L. N., Filipenko, O. S. \& Aldoshin, S. M. (1993b). Khim. Geterotsikl. Soedin. pp. 420-425.

Marthi, K., Larsen, M., Ács, M., Bálint, J. \& Fogassy, E. (1995). Acta Chem. Scand. 49, 20-27.

Marthi, K., Larsen, S., Ács, M., Bálint, J. \& Fogassy, E. (1994). Acta Cryst. B50, 762-771.

Sheldrick, G. M. (2015a). Acta Cryst. A71, 3-8.

Sheldrick, G. M. (2015b). Acta Cryst. C71, 3-8.

Spackman, M. A. \& McKinnon, J. J. (2002). CrystEngComm, 4, 378392.

Spackman, M. \& Jayatilaka, D. (2009). CrystEngComm, 11, 19-32.

Spek, A. L. (2003). J. Appl. Cryst. 36, 7-13.

Wolff, S. K., Grimwood, D. J., McKinnon, J. J., Turner, M. J., Jayatilaka, D. \& Spackman, M. A. (2012). Crystal Explorer. University of Western Australia.

Zubkov, F. I., Mertsalov, D. F., Zaytsev, V. P., Varlamov, A. V., Gurbanov, A. V., Dorovatovskii, P. V., Timofeeva, T. V., Khrustalev, V. N. \& Mahmudov, K. T. (2018). J. Mol. Liq. 249, 949-952. 


\section{supporting information}

Acta Cryst. (2019). E75, 1544-1547［https://doi.org/10.1107/S2056989019013069]

\section{Crystal structure and Hirshfeld surface analysis of 3-amino-5-phenylthia- zolidin-2-iminium bromide}

Gulnara Sh. Duruskari, Ali N. Khalilov, Mehmet Akkurt, Gunay Z. Mammadova, Taras Chyrka and Abel M. Maharramov

Computing details

Data collection: APEX2 (Bruker, 2003); cell refinement: SAINT (Bruker, 2003); data reduction: SAINT (Bruker, 2003); program(s) used to solve structure: SHELXT2014 (Sheldrick, 2015a); program(s) used to refine structure: SHELXL2016 (Sheldrick, 2015b); molecular graphics: ORTEP-3 for Windows (Farrugia, 2012); software used to prepare material for publication: PLATON (Spek, 2003).

3-Amino-5-phenylthiazolidin-2-iminium bromide

Crystal data

$\mathrm{C}_{9} \mathrm{H}_{12} \mathrm{~N}_{3} \mathrm{~S}^{+} \cdot \mathrm{Br}^{-}$

$M_{r}=274.19$

Monoclinic, $P 2_{1} / n$

$a=10.5986(5) \AA$

$b=8.7168(3) \AA$

$c=13.0308(5) \AA$

$\beta=111.513(2)^{\circ}$

$V=1119.99(8) \AA^{3}$

$Z=4$

\section{Data collection}

Bruker APEXII CCD diffractometer

$\varphi$ and $\omega$ scans

Absorption correction: multi-scan

(SADABS; Bruker, 2003)

$T_{\min }=0.534, T_{\max }=0.661$

8461 measured reflections

\section{Refinement}

Refinement on $F^{2}$

Least-squares matrix: full

$R\left[F^{2}>2 \sigma\left(F^{2}\right)\right]=0.027$

$w R\left(F^{2}\right)=0.070$

$S=1.02$

2303 reflections

127 parameters

0 restraints
$F(000)=552$

$D_{\mathrm{x}}=1.626 \mathrm{Mg} \mathrm{m}^{-3}$

Mo $K \alpha$ radiation, $\lambda=0.71073 \AA$

Cell parameters from 3357 reflections

$\theta=2.9-26.3^{\circ}$

$\mu=3.82 \mathrm{~mm}^{-1}$

$T=150 \mathrm{~K}$

Block, colorless

$0.18 \times 0.14 \times 0.11 \mathrm{~mm}$

2303 independent reflections

1998 reflections with $I>2 \sigma(I)$

$R_{\text {int }}=0.029$

$\theta_{\max }=26.4^{\circ}, \theta_{\min }=2.9^{\circ}$

$h=-13 \rightarrow 13$

$k=-10 \rightarrow 10$

$l=-16 \rightarrow 16$

Primary atom site location: structure-invariant direct methods

Secondary atom site location: difference Fourier map

Hydrogen site location: mixed

$\mathrm{H}$-atom parameters constrained

$w=1 /\left[\sigma^{2}\left(F_{\mathrm{o}}^{2}\right)+(0.0381 P)^{2}+0.5896 P\right]$

where $P=\left(F_{\mathrm{o}}{ }^{2}+2 F_{\mathrm{c}}{ }^{2}\right) / 3$ 
$(\Delta / \sigma)_{\max }=0.001$

$\Delta \rho_{\max }=0.61$ e $\AA^{-3}$

$\Delta \rho_{\min }=-0.33$ e $\AA^{-3}$

Special details

Geometry. All esds (except the esd in the dihedral angle between two 1.s. planes) are estimated using the full covariance matrix. The cell esds are taken into account individually in the estimation of esds in distances, angles and torsion angles; correlations between esds in cell parameters are only used when they are defined by crystal symmetry. An approximate (isotropic) treatment of cell esds is used for estimating esds involving l.s. planes.

Fractional atomic coordinates and isotropic or equivalent isotropic displacement parameters $\left(\AA^{2}\right)$

\begin{tabular}{|c|c|c|c|c|}
\hline & $x$ & $y$ & $z$ & $U_{\text {iso }} * / U_{\text {eq }}$ \\
\hline $\mathrm{Br} 1$ & $0.14662(3)$ & $0.38142(3)$ & $0.26547(2)$ & $0.02369(10)$ \\
\hline S1 & $0.31116(7)$ & $0.46985(7)$ & 0.58677 (5) & $0.02034(15)$ \\
\hline N1 & $0.4802(2)$ & $0.6893(2)$ & $0.61070(15)$ & $0.0159(4)$ \\
\hline $\mathrm{N} 2$ & $0.5487(2)$ & $0.8083(2)$ & $0.57955(15)$ & 0.0183 (4) \\
\hline $\mathrm{H} 2 \mathrm{~A}$ & 0.635551 & 0.776693 & 0.607350 & $0.022 *$ \\
\hline $\mathrm{H} 2 \mathrm{~B}$ & 0.535901 & 0.891753 & 0.615230 & $0.022 *$ \\
\hline N3 & $0.3577(2)$ & $0.6293(2)$ & $0.42850(16)$ & 0.0179 (4) \\
\hline $\mathrm{H} 3 \mathrm{~A}$ & 0.292719 & 0.572157 & 0.379225 & $0.021 *$ \\
\hline H3B & 0.390509 & 0.709317 & 0.402415 & $0.021 *$ \\
\hline $\mathrm{C} 1$ & $0.4372(3)$ & $0.5007(3)$ & $0.72776(19)$ & $0.0214(5)$ \\
\hline $\mathrm{H} 1 \mathrm{~A}$ & 0.513995 & 0.427032 & 0.741377 & $0.026^{*}$ \\
\hline $\mathrm{C} 2$ & $0.4890(3)$ & $0.6645(3)$ & $0.72463(19)$ & $0.0189(5)$ \\
\hline $\mathrm{H} 2 \mathrm{C}$ & 0.432008 & 0.739798 & 0.744749 & $0.023^{*}$ \\
\hline $\mathrm{H} 2 \mathrm{D}$ & 0.583832 & 0.675080 & 0.776759 & $0.023^{*}$ \\
\hline $\mathrm{C} 3$ & $0.3879(2)$ & $0.6085(3)$ & 0.53385 (19) & $0.0154(5)$ \\
\hline $\mathrm{C} 4$ & $0.3746(2)$ & $0.4760(3)$ & $0.81375(18)$ & $0.0168(5)$ \\
\hline $\mathrm{C} 5$ & $0.4280(3)$ & 0.3584 (3) & $0.8912(2)$ & $0.0212(5)$ \\
\hline $\mathrm{H} 5 \mathrm{~A}$ & 0.497585 & 0.293462 & 0.886358 & $0.025^{*}$ \\
\hline C6 & $0.3773(3)$ & $0.3379(3)$ & $0.97571(19)$ & $0.0208(5)$ \\
\hline H6A & 0.412191 & 0.258553 & 1.028582 & $0.025^{*}$ \\
\hline $\mathrm{C} 7$ & $0.2769(3)$ & $0.4330(3)$ & $0.9816(2)$ & $0.0225(5)$ \\
\hline H7A & 0.244208 & 0.420521 & 1.039905 & $0.027^{*}$ \\
\hline $\mathrm{C} 8$ & $0.2230(3)$ & $0.5463(3)$ & $0.9041(2)$ & $0.0265(6)$ \\
\hline H8A & 0.152610 & 0.610477 & 0.908407 & $0.032 *$ \\
\hline $\mathrm{C} 9$ & $0.2715(3)$ & $0.5662(3)$ & $0.8203(2)$ & $0.0249(6)$ \\
\hline H9A & 0.233208 & 0.643424 & 0.766388 & $0.030^{*}$ \\
\hline
\end{tabular}

Atomic displacement parameters $\left(\AA^{2}\right)$

\begin{tabular}{lllllll}
\hline & $U^{11}$ & $U^{22}$ & $U^{33}$ & $U^{12}$ & $U^{13}$ & $U^{23}$ \\
\hline Br1 & $0.02460(15)$ & $0.02084(15)$ & $0.02181(15)$ & $-0.00181(11)$ & $0.00401(11)$ & $-0.00181(10)$ \\
S1 & $0.0263(3)$ & $0.0175(3)$ & $0.0173(3)$ & $-0.0062(3)$ & $0.0081(2)$ & $-0.0012(2)$ \\
N1 & $0.0197(10)$ & $0.0165(10)$ & $0.0132(9)$ & $-0.0036(8)$ & $0.0078(8)$ & $-0.0004(8)$ \\
N2 & $0.0208(11)$ & $0.0162(10)$ & $0.0198(10)$ & $-0.0030(9)$ & $0.0099(9)$ & $-0.0004(8)$ \\
N3 & $0.0239(11)$ & $0.0151(10)$ & $0.0144(10)$ & $-0.0011(9)$ & $0.0066(8)$ & $-0.0014(8)$ \\
C1 & $0.0215(13)$ & $0.0233(13)$ & $0.0184(12)$ & $0.0033(11)$ & $0.0061(10)$ & $0.0023(10)$ \\
C2 & $0.0225(13)$ & $0.0215(12)$ & $0.0126(11)$ & $-0.0041(10)$ & $0.0064(10)$ & $-0.0018(10)$
\end{tabular}




\begin{tabular}{lllllll} 
C3 & $0.0176(12)$ & $0.0121(11)$ & $0.0178(12)$ & $0.0027(9)$ & $0.0082(10)$ & $-0.0009(9)$ \\
C4 & $0.0171(12)$ & $0.0193(12)$ & $0.0131(11)$ & $-0.0054(10)$ & $0.0046(9)$ & $-0.0010(9)$ \\
C5 & $0.0175(12)$ & $0.0183(12)$ & $0.0255(13)$ & $-0.0020(10)$ & $0.0051(10)$ & $-0.0066(10)$ \\
C6 & $0.0244(13)$ & $0.0193(12)$ & $0.0147(12)$ & $-0.0050(10)$ & $0.0027(10)$ & $0.0016(10)$ \\
C7 & $0.0215(13)$ & $0.0253(13)$ & $0.0214(13)$ & $-0.0100(11)$ & $0.0085(11)$ & $-0.0029(11)$ \\
C8 & $0.0208(13)$ & $0.0258(14)$ & $0.0335(15)$ & $-0.0012(11)$ & $0.0108(12)$ & $-0.0007(12)$ \\
C9 & $0.0219(13)$ & $0.0246(13)$ & $0.0266(14)$ & $0.0041(11)$ & $0.0070(11)$ & $0.0036(11)$ \\
\hline
\end{tabular}

Geometric parameters $\left(\AA,{ }^{\circ}\right)$

\begin{tabular}{|c|c|c|c|}
\hline $\mathrm{S} 1-\mathrm{C} 3$ & $1.735(2)$ & $\mathrm{C} 2-\mathrm{H} 2 \mathrm{C}$ & 0.9900 \\
\hline $\mathrm{S} 1-\mathrm{C} 1$ & $1.853(2)$ & $\mathrm{C} 2-\mathrm{H} 2 \mathrm{D}$ & 0.9900 \\
\hline $\mathrm{N} 1-\mathrm{C} 3$ & $1.318(3)$ & $\mathrm{C} 4-\mathrm{C} 9$ & $1.374(4)$ \\
\hline $\mathrm{N} 1-\mathrm{N} 2$ & $1.408(3)$ & $\mathrm{C} 4-\mathrm{C} 5$ & $1.404(3)$ \\
\hline $\mathrm{N} 1-\mathrm{C} 2$ & $1.469(3)$ & $\mathrm{C} 5-\mathrm{C} 6$ & $1.403(4)$ \\
\hline $\mathrm{N} 2-\mathrm{H} 2 \mathrm{~A}$ & 0.9000 & $\mathrm{C} 5-\mathrm{H} 5 \mathrm{~A}$ & 0.9500 \\
\hline $\mathrm{N} 2-\mathrm{H} 2 \mathrm{~B}$ & 0.9000 & $\mathrm{C} 6-\mathrm{C} 7$ & $1.373(4)$ \\
\hline $\mathrm{N} 3-\mathrm{C} 3$ & $1.303(3)$ & C6-H6A & 0.9500 \\
\hline $\mathrm{N} 3-\mathrm{H} 3 \mathrm{~A}$ & 0.9000 & $\mathrm{C} 7-\mathrm{C} 8$ & $1.378(4)$ \\
\hline N3-H3B & 0.9000 & $\mathrm{C} 7-\mathrm{H} 7 \mathrm{~A}$ & 0.9500 \\
\hline $\mathrm{C} 1-\mathrm{C} 4$ & $1.513(3)$ & $\mathrm{C} 8-\mathrm{C} 9$ & $1.378(4)$ \\
\hline $\mathrm{C} 1-\mathrm{C} 2$ & $1.535(4)$ & $\mathrm{C} 8-\mathrm{H} 8 \mathrm{~A}$ & 0.9500 \\
\hline $\mathrm{C} 1-\mathrm{H} 1 \mathrm{~A}$ & 1.0000 & $\mathrm{C} 9-\mathrm{H} 9 \mathrm{~A}$ & 0.9500 \\
\hline $\mathrm{C} 3-\mathrm{S} 1-\mathrm{C} 1$ & $91.16(11)$ & $\mathrm{N} 3-\mathrm{C} 3-\mathrm{N} 1$ & $123.6(2)$ \\
\hline $\mathrm{C} 3-\mathrm{N} 1-\mathrm{N} 2$ & $119.48(18)$ & $\mathrm{N} 3-\mathrm{C} 3-\mathrm{S} 1$ & $123.08(18)$ \\
\hline $\mathrm{C} 3-\mathrm{N} 1-\mathrm{C} 2$ & $116.3(2)$ & $\mathrm{N} 1-\mathrm{C} 3-\mathrm{S} 1$ & $113.33(17)$ \\
\hline $\mathrm{N} 2-\mathrm{N} 1-\mathrm{C} 2$ & $123.48(18)$ & $\mathrm{C} 9-\mathrm{C} 4-\mathrm{C} 5$ & $119.6(2)$ \\
\hline $\mathrm{N} 1-\mathrm{N} 2-\mathrm{H} 2 \mathrm{~A}$ & 102.6 & $\mathrm{C} 9-\mathrm{C} 4-\mathrm{C} 1$ & $122.7(2)$ \\
\hline $\mathrm{N} 1-\mathrm{N} 2-\mathrm{H} 2 \mathrm{~B}$ & 104.8 & $\mathrm{C} 5-\mathrm{C} 4-\mathrm{C} 1$ & $117.7(2)$ \\
\hline $\mathrm{H} 2 \mathrm{~A}-\mathrm{N} 2-\mathrm{H} 2 \mathrm{~B}$ & 111.4 & $\mathrm{C} 6-\mathrm{C} 5-\mathrm{C} 4$ & $119.2(2)$ \\
\hline $\mathrm{C} 3-\mathrm{N} 3-\mathrm{H} 3 \mathrm{~A}$ & 120.2 & $\mathrm{C} 6-\mathrm{C} 5-\mathrm{H} 5 \mathrm{~A}$ & 120.4 \\
\hline $\mathrm{C} 3-\mathrm{N} 3-\mathrm{H} 3 \mathrm{~B}$ & 121.7 & $\mathrm{C} 4-\mathrm{C} 5-\mathrm{H} 5 \mathrm{~A}$ & 120.4 \\
\hline $\mathrm{H} 3 \mathrm{~A}-\mathrm{N} 3-\mathrm{H} 3 \mathrm{~B}$ & 117.4 & $\mathrm{C} 7-\mathrm{C} 6-\mathrm{C} 5$ & $119.6(2)$ \\
\hline $\mathrm{C} 4-\mathrm{C} 1-\mathrm{C} 2$ & $114.2(2)$ & $\mathrm{C} 7-\mathrm{C} 6-\mathrm{H} 6 \mathrm{~A}$ & 120.2 \\
\hline $\mathrm{C} 4-\mathrm{C} 1-\mathrm{S} 1$ & $111.16(17)$ & $\mathrm{C} 5-\mathrm{C} 6-\mathrm{H} 6 \mathrm{~A}$ & 120.2 \\
\hline $\mathrm{C} 2-\mathrm{C} 1-\mathrm{S} 1$ & $104.09(16)$ & $\mathrm{C} 6-\mathrm{C} 7-\mathrm{C} 8$ & $120.9(2)$ \\
\hline $\mathrm{C} 4-\mathrm{C} 1-\mathrm{H} 1 \mathrm{~A}$ & 109.1 & $\mathrm{C} 6-\mathrm{C} 7-\mathrm{H} 7 \mathrm{~A}$ & 119.5 \\
\hline $\mathrm{C} 2-\mathrm{C} 1-\mathrm{H} 1 \mathrm{~A}$ & 109.1 & $\mathrm{C} 8-\mathrm{C} 7-\mathrm{H} 7 \mathrm{~A}$ & 119.5 \\
\hline $\mathrm{S} 1-\mathrm{C} 1-\mathrm{H} 1 \mathrm{~A}$ & 109.1 & $\mathrm{C} 9-\mathrm{C} 8-\mathrm{C} 7$ & $119.7(3)$ \\
\hline $\mathrm{N} 1-\mathrm{C} 2-\mathrm{C} 1$ & $105.85(19)$ & $\mathrm{C} 9-\mathrm{C} 8-\mathrm{H} 8 \mathrm{~A}$ & 120.1 \\
\hline $\mathrm{N} 1-\mathrm{C} 2-\mathrm{H} 2 \mathrm{C}$ & 110.6 & $\mathrm{C} 7-\mathrm{C} 8-\mathrm{H} 8 \mathrm{~A}$ & 120.1 \\
\hline $\mathrm{C} 1-\mathrm{C} 2-\mathrm{H} 2 \mathrm{C}$ & 110.6 & $\mathrm{C} 4-\mathrm{C} 9-\mathrm{C} 8$ & $120.9(2)$ \\
\hline $\mathrm{N} 1-\mathrm{C} 2-\mathrm{H} 2 \mathrm{D}$ & 110.6 & $\mathrm{C} 4-\mathrm{C} 9-\mathrm{H} 9 \mathrm{~A}$ & 119.5 \\
\hline $\mathrm{C} 1-\mathrm{C} 2-\mathrm{H} 2 \mathrm{D}$ & 110.6 & $\mathrm{C} 8-\mathrm{C} 9-\mathrm{H} 9 \mathrm{~A}$ & 119.5 \\
\hline $\mathrm{H} 2 \mathrm{C}-\mathrm{C} 2-\mathrm{H} 2 \mathrm{D}$ & 108.7 & & \\
\hline $\mathrm{C} 3-\mathrm{S} 1-\mathrm{C} 1-\mathrm{C} 4$ & $147.17(19)$ & $\mathrm{C} 2-\mathrm{C} 1-\mathrm{C} 4-\mathrm{C} 9$ & $53.9(3)$ \\
\hline
\end{tabular}




\begin{tabular}{llll}
$\mathrm{C} 3-\mathrm{S} 1-\mathrm{C} 1-\mathrm{C} 2$ & $23.78(17)$ & $\mathrm{S} 1-\mathrm{C} 1-\mathrm{C} 4-\mathrm{C} 9$ & $-63.5(3)$ \\
$\mathrm{C} 3-\mathrm{N} 1-\mathrm{C} 2-\mathrm{C} 1$ & $26.7(3)$ & $\mathrm{C} 2-\mathrm{C} 1-\mathrm{C} 4-\mathrm{C} 5$ & $-124.2(2)$ \\
$\mathrm{N} 2-\mathrm{N} 1-\mathrm{C} 2-\mathrm{C} 1$ & $-163.3(2)$ & $\mathrm{S} 1-\mathrm{C} 1-\mathrm{C} 4-\mathrm{C} 5$ & $118.4(2)$ \\
$\mathrm{C} 4-\mathrm{C} 1-\mathrm{C} 2-\mathrm{N} 1$ & $-152.1(2)$ & $\mathrm{C} 9-\mathrm{C} 4-\mathrm{C} 5-\mathrm{C} 6$ & $-1.7(4)$ \\
$\mathrm{S} 1-\mathrm{C} 1-\mathrm{C} 2-\mathrm{N} 1$ & $-30.7(2)$ & $\mathrm{C} 1-\mathrm{C} 4-\mathrm{C} 5-\mathrm{C} 6$ & $176.5(2)$ \\
$\mathrm{N} 2-\mathrm{N} 1-\mathrm{C} 3-\mathrm{N} 3$ & $1.9(3)$ & $\mathrm{C} 4-\mathrm{C} 5-\mathrm{C} 6-\mathrm{C} 7$ & $-0.2(4)$ \\
$\mathrm{C} 2-\mathrm{N} 1-\mathrm{C} 3-\mathrm{N} 3$ & $172.3(2)$ & $\mathrm{C} 5-\mathrm{C} 6-\mathrm{C} 7-\mathrm{C} 8$ & $1.5(4)$ \\
$\mathrm{N} 2-\mathrm{N} 1-\mathrm{C} 3-\mathrm{S} 1$ & $-178.62(16)$ & $\mathrm{C} 6-\mathrm{C} 7-\mathrm{C} 8-\mathrm{C} 9$ & $-0.9(4)$ \\
$\mathrm{C} 2-\mathrm{N} 1-\mathrm{C} 3-\mathrm{S} 1$ & $-8.2(3)$ & $\mathrm{C} 5-\mathrm{C} 4-\mathrm{C} 9-\mathrm{C} 8$ & $2.2(4)$ \\
$\mathrm{C} 1-\mathrm{S} 1-\mathrm{C} 3-\mathrm{N} 3$ & $169.2(2)$ & $\mathrm{C} 1-\mathrm{C} 4-\mathrm{C} 9-\mathrm{C} 8$ & $-175.8(2)$ \\
$\mathrm{C} 1-\mathrm{S} 1-\mathrm{C} 3-\mathrm{N} 1$ & $-10.29(19)$ & $\mathrm{C} 7-\mathrm{C} 8-\mathrm{C} 9-\mathrm{C} 4$ & $-1.0(4)$ \\
\hline
\end{tabular}

Hydrogen-bond geometry $\left(A,{ }^{\circ}\right)$

\begin{tabular}{lllll}
\hline$D-\mathrm{H} \cdots A$ & $D-\mathrm{H}$ & $\mathrm{H} \cdots A$ & $D \cdots A$ & $D-\mathrm{H} \cdots A$ \\
\hline $\mathrm{N} 2-\mathrm{H} 2 A \cdots \mathrm{Br} 1^{\mathrm{i}}$ & 0.90 & 2.68 & $3.530(2)$ & 158 \\
$\mathrm{~N} 2-\mathrm{H} 2 B \cdots \mathrm{Br} 1^{\mathrm{ii}}$ & 0.90 & 2.73 & $3.524(2)$ & 148 \\
$\mathrm{~N} 3-\mathrm{H} 3 A \cdots \mathrm{Br} 1$ & 0.90 & 2.38 & $3.271(2)$ & 169 \\
$\mathrm{~N} 3-\mathrm{H} 3 B \cdots \mathrm{Br} 1^{\mathrm{iii}}$ & 0.90 & 2.56 & $3.337(2)$ & 145 \\
\hline
\end{tabular}

Symmetry codes: (i) $-x+1,-y+1,-z+1$; (ii) $x+1 / 2,-y+3 / 2, z+1 / 2$; (iii) $-x+1 / 2, y+1 / 2,-z+1 / 2$. 